\title{
Blocking Probabilities In A Loss System with Arrivals in Geometrically Distributed Batches and Heterogeneous Service Requirements
}

\author{
Erik A. van Doorn and Frans J. M. Panken
}

\begin{abstract}
We analyze a generalization of the classical Erlang loss model. Customers of several types contend for access to a service facility consisting of a finite number of servers. Each customer requires a fixed number of servers simultaneously during an exponentially distributed service time, and is blocked on arrival if this requirement cannot be met. Customers of each type arrive in geometrically distributed batches, while the arrival of batches of each type is governed by a Poisson process. All relevant parameters may be type-dependent. We obtain the steady-state distribution of the number of customers of each type in the system (which turns out to have product form), and the blocking probabilities experienced by each customer type. In addition, we bring to light the connection between the model at hand and a method proposed by Delbrouck for estimating blocking probabilities in an incompletely specified setting.
\end{abstract}

\section{INTRODUCTION}

W E CONSIDER a system consisting of $N$ service units at which $K$ independent streams of customers arrive. Customers in stream $i$ (which will be called type- $i$ customers) require $c_{i}$ service units simultaneously during an exponentially distributed time of mean $\mu_{i}^{-1}, i=1,2, \cdots, K$. Customers who find on arrival that their requirements cannot be met disappear from the system and are said to be blocked.

When customers in each stream arrive according to a Poisson process we are dealing with a model which has been studied quite extensively. In particular, efficient procedures have been developed for computing the distribution of the number of occupied units and the blocking probabilities experienced by the individual streams, see [5], [7], [9] and the references there. Salient features of the model at hand are the product form of the solution of the Markovian state equations and its insensitivity to service time distributions apart from their means.

In this note we discuss a generalization of the above model in which type- $i$ customers arrive in batches of size $b_{i}$, where

$$
\operatorname{Pr}\left\{b_{i}=j\right\}=\left(1-r_{i}\right) r_{i}^{j-1}, \quad j=1,2, \ldots,
$$

and $0 \leq r_{i}<1$, while the arrival of batches is governed by a Poisson process with intensity $\nu_{i}$. The customers in a batch

Manuscript received September 1992; revised June 1993; approved by IEEE/ACM Transactions on Networking Editor D. Mitra.

E. van Doorn is with the Faculty of Applied Mathematics, University of Twente, Enschede, The Netherlands (email: doom@math.utwente.nl)

F. Panken is with the Dept. of Computer Science, University of Nijmegen, Nijmegen, The Netherlands (email: fransp@cs.kun.nl).

IEEE Log Number 9215400. are ordered randomly and dealt with individually. Hence, if a batch cannot be accommodated completely, the part which can be fitted is allowed in and the rest is discarded. Surprisingly, several features of the model in which customers arrive singly are maintained in this generalization. In particular, the Markovian state equations have a product-form solution, and there exist efficient methods for computing the state and blocking probabilities, as will be shown in the Sections II and III.

Apart from its interest per se our generalization to batch arrivals with geometrically distributed batch sizes is of interest because it gives us a model which fits in a setting where the arrival streams are characterized by their means and peakedness factors $(\geq 1)$ only. Indeed, it will be shown in Section IV that this fitting approach leads to an algorithm which is completely identical to Delbrouck's [2] scheme for estimating blocking probabilities in such a setting. As an aside we remark that the original idea of fitting a batch Poisson process with geometrically distributed batch sizes in a setting where only mean and peakedness factor are known, seems to be due to Jensen [6].

As Delbrouck's paper [2] generalizes his earlier paper [1] to heterogeneous service requirements, so constitutes the present paper a generalization of [3], which deals with the special case of homogeneous service requirements, that is, $c_{i}=1$ and $\mu_{i}=\mu$ for all $i$.

\section{STEADY-STATE PROBABILITIES}

The state of the system will be represented by the vector $\boldsymbol{x} \equiv\left(x_{1}, x_{2}, \cdots, x_{K}\right)$, where $x_{i}$ denotes the number of type- $i$ customers in the service system. The set of all possible states is denoted by $\Omega$, that is,

$$
\Omega \equiv\left\{\boldsymbol{x} \equiv\left(x_{1}, x_{2}, \cdots, x_{K}\right) \mid \sum_{i=1}^{K} c_{i} x_{i} \leq N\right\} .
$$

It will be convenient to introduce the notation

$$
\boldsymbol{x}_{i}^{ \pm j} \equiv\left(x_{1}, \cdots, x_{i-1}, x_{i} \pm j, x_{i+1}, \cdots, x_{K}\right),
$$

and

$$
\delta_{i}(x) \equiv\left\{\begin{array}{ll}
1 & \text { if } x_{i}^{+1} \in \Omega \\
0 & \text { otherwise }
\end{array} .\right.
$$

With these conventions, and with $p(x)$ denoting the steadystate probability that the system is in state $x$, the balance 
equations for the $K$-dimensional Markov chain representing the state of the system can be written down at once as

$$
\begin{aligned}
& \left\{\sum_{i=1}^{K}\left(\nu_{i} \delta_{i}(\boldsymbol{x})+\mu_{i} x_{i}\right)\right\} p(x)= \\
& \sum_{i=1}^{K} \mu_{i}\left(x_{i}+1\right) \delta_{i}(\boldsymbol{x}) p\left(\boldsymbol{x}_{i}^{+1}\right)+ \\
& +\sum_{i=1}^{K} \sum_{j=1}^{x_{i}} \nu_{i}\left(1-r_{i} \delta_{i}(\boldsymbol{x})\right) r_{i}^{j-1} p\left(\boldsymbol{x}_{i}^{-j}\right), \quad x \in \Omega .
\end{aligned}
$$

Theorem 1: The steady-state distribution is given by

$$
p(\boldsymbol{x})=(G(\Omega))^{-1} \prod_{i=1}^{K} p_{i}\left(x_{i}\right), \quad x \in \Omega
$$

where

$$
p_{i}(x) \equiv\left(\begin{array}{c}
\varrho_{i}+x-1 \\
x
\end{array}\right) r_{i}^{x}=\frac{\Gamma\left(\varrho_{i}+x\right)}{\Gamma\left(\varrho_{i}\right)} \frac{r_{i}^{x}}{x !}, \quad x=0,1, \ldots
$$

with

$$
\varrho_{i} \equiv \nu_{i} /\left(\mu_{i} r_{i}\right)
$$

and

$$
G(\Omega) \equiv \sum_{x \in \Omega} \prod_{i=1}^{K} p_{i}\left(x_{i}\right)
$$

Proof: It is easily seen that if $p(x), x \in \Omega$, satisfies (2) - (5), then, for $i=1,2, \cdots, K$,

$$
\mu_{i}\left(x_{i}+1\right) \delta_{i}(\boldsymbol{x}) p\left(\boldsymbol{x}_{i}^{+1}\right)=\left(\nu_{i}+r_{i} \mu_{i} x_{i}\right) \delta_{i}(\boldsymbol{x}) p(\boldsymbol{x})
$$

and, for $j=1,2, \cdots, x_{i}$,

$$
\nu_{i} r_{i}^{j-1} p\left(x_{i}^{-j}\right)=\mu_{i} \varrho_{i}\left(\begin{array}{c}
x_{i} \\
j
\end{array}\right)\left(\begin{array}{c}
\varrho_{i}+x_{i}-1 \\
j
\end{array}\right)^{-1} p(\boldsymbol{x}) .
$$

Exploiting the identity

$$
\sum_{j=1}^{n}\left(\begin{array}{l}
n \\
j
\end{array}\right)\left(\begin{array}{c}
\varrho+n-1 \\
j
\end{array}\right)^{-1}=\frac{n}{\varrho}
$$

see, e.g., [8, p. 154], it subsequently follows that $p(x), x \in \Omega$, satisfies the partial balance equations

$$
\begin{aligned}
& \left(\nu_{i} \delta_{i}(\boldsymbol{x})+\mu_{i} x_{i}\right) p(\boldsymbol{x})= \\
& \mu_{i}\left(x_{i}+1\right) \delta_{i}(\boldsymbol{x}) p\left(\boldsymbol{x}_{i}^{+1}\right)+ \\
& +\sum_{j=1}^{x_{i}} \nu_{i}\left(1-r_{i} \delta_{i}(\boldsymbol{x})\right) r_{i}^{j-1} p\left(\boldsymbol{x}_{i}^{-j}\right), \quad x \in \Omega,
\end{aligned}
$$

and hence the global balance equations (1).

We note that, apart from the normalizing factor (1 $\left.r_{i}\right)^{\varrho_{i}}$, the quantities $p_{i}(x), x=0,1, \ldots$ constitute a negative binomial distribution with parameters $\varrho_{i}$ and $1-r_{i}$. Since $\Gamma(\varrho+x) / \Gamma(\varrho) \sim \varrho^{x}$ for $\varrho \rightarrow \infty$, we have

$$
\lim _{r \downarrow 0}\left(\begin{array}{c}
\nu /(\mu r)+x-1 \\
x
\end{array}\right) r^{x}=\frac{(\nu / \mu)^{x}}{x !}
$$

so that Theorem 1 reduces to the well-known result for single arrivals if we let $r_{i} \downarrow 0$ for all $i$.
The efficient algorithm developed in [7] and [9] for computing the steady-state distribution of the total number of occupied service units in the case of single arrivals is based on a recurrence relation. This relation (as well as the algorithm) can be generalized to the present context, as can be seen from the next theorem. We let $q(j)$ denote the probability that the number of occupied service units in steady state equals $j$, $j=0,1, \cdots, N$, and $q(j) \equiv 0$ for $j<0$; also, for any real number $a,[a]$ denotes the largest integer less than or equal to $a$.

Theorem 2: The steady-state probabilities $q(j), j=$ $0,1, \cdots, N$, satisfy the relations

$$
j q(j)=\sum_{i=1}^{K} \frac{c_{i} \nu_{i}}{\mu_{i}} \sum_{m=1}^{\left[j / c_{i}\right]} r_{i}^{m-1} q\left(j-m c_{i}\right), \quad j=1,2, \cdots, N
$$

and

$$
\sum_{j=0}^{N} q(j)=1
$$

Proof: We have

$$
q(j)=\sum_{x \in \Omega_{j}} p(x), \quad j=0,1, \cdots, N,
$$

where

$$
\Omega_{j} \equiv\left\{x \equiv\left(x_{1}, \cdots, x_{K}\right) \mid \sum_{i=1}^{K} x_{i} c_{i}=j\right\} .
$$

Hence, for $j>0$,

$$
\begin{aligned}
j q(j) & =\sum_{i=1}^{K} c_{i} \sum_{x \in \Omega_{j}} x_{i} p(\boldsymbol{x}) \\
& =\sum_{i=1}^{K} c_{i} \sum_{\boldsymbol{x} \in \Omega_{j}} \varrho_{i} \sum_{m=1}^{x_{i}}\left(\begin{array}{c}
x_{i} \\
m
\end{array}\right)\left(\begin{array}{c}
\varrho_{i}+x_{i}-1 \\
m
\end{array}\right)^{-1} p(\boldsymbol{x}) \\
& =\sum_{i=1}^{K}\left(c_{i} \nu_{i} / \mu_{i}\right) \sum_{\boldsymbol{x} \in \Omega_{j}} \sum_{m=1}^{x_{i}} r_{i}^{m-1} p\left(\boldsymbol{x}_{i}^{-m}\right) \\
& =\sum_{i=1}^{K}\left(c_{i} \nu_{i} / \mu_{i}\right) \sum_{m=1}^{\left[j / c_{i}\right]} \sum_{\boldsymbol{x} \in \Omega_{j-m c_{i}}} r_{i}^{m-1} p(\boldsymbol{x}) \\
& =\sum_{i=1}^{K}\left(c_{i} \nu_{i} / \mu_{i}\right) \sum_{m=1}^{\left[j / c_{i}\right]} r_{i}^{m-1} q\left(j-m c_{i}\right)
\end{aligned}
$$

where we have used (7) and (8)

\section{BLocking Probabilities}

We will now express the steady-state blocking probabilities $B_{i}$ experienced by type- $i$ customers, $i=1,2, \cdots, K$, in terms of the probabilities $q(j)$ of the previous section.

Exploiting PASTA (Poisson Arrivals See Time Averages) we notice that the stream of blocked type- $i$ customers constitutes a batch Poisson process in which the arrival rate of 
batches is $\nu_{i}$ (as in the original stream), but the batch size $\tilde{b}_{i}$ is distributed as

$$
\operatorname{Pr}\left\{\tilde{b}_{i}=j\right\}=\left\{\begin{array}{ll}
\sum_{m=0}^{N-1} q(m)\left(1-r_{i}^{\left[\frac{N-m}{c_{i}}\right]}\right) & \text { if } j=0 \\
\left(1-r_{i}\right) \sum_{m=0}^{N} q(m) r_{i}^{\left[\frac{N-m}{c_{i}}\right]+j-1} & \text { if } j>0
\end{array} .\right.
$$

It follows that the average batch size is given by

$$
E \tilde{b}_{i}=\sum_{j=0}^{\infty} j \operatorname{Pr}\left\{\tilde{b}_{i}=j\right\}=\left(1-r_{i}\right)^{-1} \sum_{m=0}^{N} q(m) r_{i}^{\left[(N-m) / c_{i}\right]} \text {. }
$$

Since $B_{i}=E \tilde{b}_{i} / E b_{i}$, while $E b_{i}=\left(1-r_{i}\right)^{-1}$, the next result emerges.

Theorem 3 The steady-state blocking probability $B_{i}$ experienced by type- $i$ customers satisfies

$$
B_{i}=\sum_{m=0}^{N} q(m) r_{i}^{\left[(N-m) / c_{i}\right]}, \quad i=1,2, \cdots, K .
$$

We note that the time congestion $C_{i}$ for type- $i$ customers is given by

$$
C_{i}=\sum_{m=N-c_{i}+1}^{N} q(m)
$$

Hence, letting $r_{i} \downarrow 0$ in (10) leads to $B_{i}=C_{i}$ which, by PASTA, is indeed the result prevailing when arrivals occur singly.

\section{Concluding Remarks}

Defining $m_{i}$ and $v_{i}$ as the mean and variance, respectively, of the number of type- $i$ customers in the system when $N=\infty$, it is easily seen that

$$
m_{i}=\left(1-r_{i}\right)^{-1} \nu_{i} / \mu_{i}
$$

and

$$
z_{i} \equiv v_{i} / m_{i}=\left(1-r_{i}\right)^{-1} \text {. }
$$

The quantities $m_{i}$ and $z_{i}$ are called the mean and peakedness factor, respectively, of stream $i$.

Now suppose that we are dealing with a system in which the arrival streams have known means $m_{i}$ and peakedness factors $z_{i} \geq 1$, but are otherwise unspecified. From (11) and (12) it then follows that by choosing

$$
r_{i} \equiv 1-z_{i}^{-1}
$$

and

$$
\nu_{i} \equiv \mu_{i} m_{i} z_{i}^{-1}
$$

we can fit our batch Poisson model of the previous sections into this setting. Subsequently, we can obtain estimates for the blocking probabilities experienced by each stream in the original, incompletely specified model by applying the results of the previous sections. Surprisingly however, a little algebra reveals the equivalence of (9) and (10) on the one hand, and a scheme proposed by Delbrouck [2] for estimating blocking probabilities in this partially specified setting on the other hand. So we have shown that for given means $m_{i}$ and peakedness factors $z_{i} \geq 1, i=1,2, \cdots, K$, arrival streams can be constructed, which, when offered to a common system, experience blocking probabilities that are exactly determined by Delbrouck's recursive scheme. Upon some reflection this result is less surprising than it seems at first sight, since the assumption underlying Delbrouck's scheme is essentially the system of balance equations (6), which is equivalent to the system (1).

Finally we remark that Delbrouck's estimative scheme has been generalized to fixed-routing circuit-switched networks by Dziong and Roberts [4]. It is not difficult to see that our interpretation of Delbrouck's scheme in the single-station case can also be generalized to this wider context.

\section{ACKNOWLEDGMENT}

The authors would like to thank André Roosma, Bart Sanders, Peter Taylor, and the referees for their comments and suggestions.

\section{REFERENCES}

[1] L. E. N. Delbrouck, "The uses of Kosten's systems in the provisioning of altemate trunk groups carrying heterogeneous traffic," IEEE Trans. Commun., vol. COM-31, pp. 741-749, 1983.

[2] L. E. N. Delbrouck, "On the steady-state distribution in a service facility carrying mixtures of traffic with different peakedness factors and capacity requirements," IEEE Trans. Commun., vol. COM-31, pp. 1209-1211, 1983.

[3] E. A. van Doorn, "A note on Delbrouck's approximate solution to the heterogeneous blocking problem," IEEE Trans. Commun., vol. COM-32, pp. $1210-1211,1984$.

[4] Z. Dziong and J. W. Roberts, "Congestion probabilities in a circuitswitched integrated services network," Performance Evaluation, vol. 7 , pp. 267-284, 1987

[5] V. B. Iversen, "The exact evaluation of multi-service loss systems with access control," Teleteknik(English), vol. 31, pp. 56-61, 1987.

[6] E. Jensen, "Discussion on A. H. Freeman's paper 'Accuracy of overflow traffic models," Austral. Telecommun. Res., vol. 11, p. 103, 1977.

[7] J. S. Kaufman, "Blocking in a shared resource environment,",IEEE Trans. Commun., vol. COM-29, pp. $1474-1481,1981$.

[8] F. P. Kelly, Reversibility and Stochastic Networks. Chichester, United Kingdom: Wiley, 1979.

[9] J. W. Roberts, "A service system with heterogeneous user requirements-Application to multiservice telecommunications systems," in Performance of Data Communications Systems and Their Applications, G. Pujolle, ed. New York: North Holland, 1981, pp. 423-431.

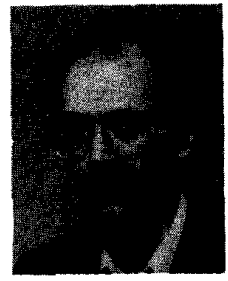

Erik A. van Doorn received the M. Sc. degree from Eindhoven University of Technology in 1974 and the $\mathrm{Ph}$. $\mathrm{D}$. degree from the University of Twente, Enschede, The Netherlands, in 1979.

Since 1985 he has been an Associate Professor at the Faculty of Applied Mathematics of the University of Twente. His research interests range from theoretical issues in mathematical analysis and probability theory to performance modeling and analysis of computer and communication systems.

Dr. van Doorn serves on the editorial boards of Telecommunication Systems and Stochastic Models. 


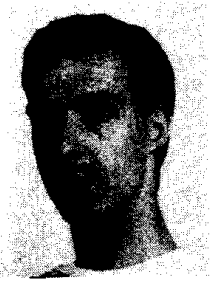

Frans J. M. Panken received the M. Sc. degree in mathematics from the University of Twente, Enschede, The Netherlands, in 1992.

Since 1992, he has been working as a researcher at the Dept. of Computer Science of the University of Nijmegen, The Netherlands. His research interests include stochastic modeling and performance analysis of computer systems and communication networks. 